\title{
Características sociodemográficas y clínicas de usuarios de un centro de rehabilitación de adicciones
}

\author{
Daniela Romero Reyes', José Luis Ybarra Sagarduy', Luz Adriana Orozco Ramírez' \\ I Universidad Autónoma de Tamaulipas, México
}

\begin{abstract}
RESUMEN
Introducción: las adicciones representan un serio problema de salud pública. Existe la necesidad de realizar más estudios sobre las características de los consumidores de drogas en general para desarrollar tratamientos más específicos. Objetivo: el objetivo de este estudio fue identificar las características sociodemográficas/ clínicas de usuarios de un Centro de Rehabilitación de adicciones y establecer posibles asociaciones entre perfiles de personalidad y droga de impacto. Método: el estudio fue no experimental, descriptivo y correlacional; la muestra estuvo conformada por 300 participantes, en su mayoría varones solteros, estudiantes de bachillerato y licenciatura. Resultados: las principales drogas de elección reportadas fueron alcohol, mariguana y cocaína. Se encontró mayor cantidad de usuarios con rasgos de personalidad antisocial, seguidos por rasgos de personalidad límite y esquizoide. Discusión y conclusiones: se observa la necesidad de prevención primaria y secundaria. La rehabilitación es una opción favorable y necesaria para la recuperación de usuarios y sus familias.
\end{abstract}

Palabras clave: adicciones, tratamiento, rehabilitación, características sociodemográficas, perfil psicológico.

\begin{abstract}
Introduction: addictions represent a serious public health problem. There is a need to conduct more studies on the characteristics of drug users in general to develop more specific treatments. Objective: the objective of this study was to identify the sociodemographic/clinical characteristics of users of a Center for the Rehabilitation of addictions and to establish possible associations between personality profiles and drugs. Method: the study was non-experimental, descriptive and correlational, the sample consisted of 300 participants, mostly single males, high school and undergraduate students. Results: the main drugs of choice reported were alcohol, marijuana and cocaine. A greater number of users with antisocial personality traits were found, followed by borderline and schizoid personality traits. Discussion and conclusion: the need for primary and secondary prevention is observed. Rehabilitation is a favorable and necessary option for the recovery of users and their families.
\end{abstract}

Keywords: addictions, treatment, rehabilitation, sociodemographic characteristics, psychological profile

\footnotetext{
Autor de correspondencia:

Daniela Romero Reyes. Tenochtitlán esq. Cuicuilco núm. 136, Fracc. México, Cd. Victoria, Tamaulipas, México. C.P. 87049. Tel.: 04483430167 27. Correo electrónico: danielaromeroreyes@gmail.com

Recibido: 17 de septiembre de 2018

Aceptado: 26 de marzo de 2019

doi: 10.28931/riiad.2019.1.04
} 


\section{INTRODUCCIÓN}

Las adicciones representan un grave problema de salud pública. El Reporte Anual de la Oficina de las Naciones Unidas contra la Droga y el Delito (UNODC, 2017) estima que se perdieron 28 millones de años de vida sana en todo el mundo durante el 2015 relacionados con discapacidad y muerte prematura por consumo de drogas; sin embargo, la oferta de tratamientos y el acceso a ellos continúa limitado, pues sólo una de cada seis personas que lo necesitan reciben tratamiento al año.

La Encuesta Nacional de Consumo de Drogas, Alcohol y Tabaco, Reporte de drogas de México (ENCODAT, Villatoro et al., 2017a) indica que $0.6 \%$ de los participantes presenta posible dependencia del consumo de drogas y $2.2 \%$ dependencia del alcohol (Villatoro-Velázquez et al., 2017b). Los dependientes de drogas con edades de 18 a 35 años presentaron mayor número de días perdidos por el consumo durante los últimos 12 meses (23 días), lo que deja entrever las consecuencias de las adicciones en el ámbito productivo. Además, el consumo de drogas se ha incrementado en las mujeres (Villatoro-Velázquez et al., 2017a).

Para la Norma Oficial Mexicana (NOM) 028 (Secretaría de Salud, 2009), el tratamiento se refiere a las estrategias para obtener la abstinencia o reducir el consumo y los riesgos concomitantes y fomentar el bienestar integral; el tratamiento especializado en adicciones puede ser ambulatorio o residencial. Los tratamientos residenciales se efectúan en establecimientos que pueden operar bajo un modelo profesional, de ayuda mutua o mixta. Los tratamientos profesionales son ofrecidos por especialistas de la salud. Los tratamientos de ayuda mutua son operados por grupos de personas recuperadas de su dependencia del alcohol o drogas y utilizan programas de autoayuda como Alcohólicos Anónimos. Los tratamientos mixtos proporcionan sus servicios con equipos integrados por profesionales de la salud y personal en recuperación que cuenta con la debida preparación y proceso de rehabilitación satisfactorio, con años de demostrada abstinencia. En la NOM 028 se especifican los elementos indispensables para la operación de los centros, los cuales deben contar con instalaciones adecuadas, expediente clínico, revisión del caso por el médico responsable y equipo clínico, diagnóstico, tratamiento, notas de evolución y resumen de egreso; además, debe llevarse a cabo vigilancia epidemiológica, por lo que es obligatorio que los centros reporten al órgano oficial que genera información actualizada del comportamiento de las adicciones en el país: el Sistema de Vigilancia Epidemiológica en Adicciones (SISVEA; Secretaría de Salud, 2016).

EI SISVEA complementa la información que existe en materia de consumo de sustancias y para ello analiza la información de Centros de Tratamiento y Rehabilitación No Gubernamentales, Consejos Tutelares de Menores, Servicio Médico Forenses (SEMEFO) y Servicios de Urgencias Hospitalarias. Durante 2016, el SISVEA reportó 60,582 nuevos ingresos a tratamiento, en los 32 estados de la república, que acudieron a alguno de los 1,125 Centros de Tratamiento ubicados en el país. De acuerdo con este informe, en Tamaulipas la cocaína fue la principal droga de impacto consumida (droga que causa mayores problemas en la vida del usuario y por la que decide ir a rehabilitación).

Si bien la información epidemiológica existente en México es necesaria para el diseño de estrategias de prevención y tratamiento, es fundamental llevar registros del patrón de crecimiento de la demanda de atención a los servicios de salud especializados en el tratamiento de las adicciones, los cuales aún son escasos a pesar de las aportaciones de los sistemas oficiales existentes (Diaz-Negrete, Gutiérrez-López, Fernández-Cáceres, \& Sánchez-Huesca, 2015).

Además de conocer la demanda de atención de centros especializados en el tratamiento de las adicciones, se vuelve relevante describir las características de las personas que solicitan ayuda. En este sentido, Cázares et al. (2010) describieron a usuarios de una comunidad terapéutica e internos de una unidad terapéutica intra-penitanciaria, encontraron muestras compuestas mayormente por hombres solteros, con baja escolaridad y con algún problema médico crónico; la cocaína fue la droga de consumo principal en ambos grupos; también se encontró como antecedente el primer contacto de la droga principal en la adolescencia; además, el grupo de personas en prisión presentó mayor alteración en los rasgos de personalidad y más trastornos de personalidad graves. Capistrano, Ferreira, Silva, Kalinke y Maftum (2013), en un estudio realizado en Brasil con el objetivo de caracterizar a 350 usuarios de una unidad de rehabilitación de un hospital psiquiátrico, encontraron participantes con una media de edad de 35.8 años, que habían comenzado el consumo en la adolescencia y poco más del $50 \%$ era dependiente del alcohol, $45.1 \%$ se encontraba desempleado, $13 \%$ tuvo comorbilidad psiquiátrica y $30.1 \%$ comorbilidades clínicas. Otro estudio realizado en México por Oropeza, Lira y Rosales (2013) buscó conocer el perfil sociodemográfico y patrón de uso de cocaína y crack y su asociación con otras drogas. La muestra total fue de 139 usuarios del Centro de Prevención y Atención en Adicciones de la Universidad Nacional Autónoma de México (UNAM). Se encontró mayor proporción de hombres, los consumidores de cocaína tuvieron mayor escolaridad que los de crack, $21 \%$ no trabajaba ni estudiaba, 54.4\% recibió tratamiento previo, los consumidores de cocaína tuvie- 
ron más años de consumo que los de crack, el consumo de alcohol y tabaco concomitante a la cocaína y al crack fue elevado.

Otros estudios con dependientes de sustancias han abordado el tema de la personalidad. Al respecto, Carou, Romero y Luengo (2013) señalan que estas investigaciones han brindado una valiosa información para comprender las adicciones. La personalidad, ya sea normal o patológica, en esencia contiene los mismos rasgos (Cardenal, Sánchez, \& Ortiz-Tallo, 2007). Para la evaluación multiaxial, en la personalidad se originan las conductas desadaptativas, por lo que es sumamente importante comprender de manera integral a la persona y contextualizar los síntomas en el panorama de la personalidad (Cardenal, Sánchez, \& Ortiz-Tallo, 2007), lo cual puede ser beneficioso para los servicios de salud, al favorecer la individualización de los tratamientos.

Oropeza et al. (2013) indican que existe la necesidad de realizar más estudios acerca de las características de los consumidores de drogas en general, pues dicho conocimiento puede ayudar a desarrollar tratamientos más acordes con sustancias específicas; asimismo, este tipo de investigaciones aportan información relevante para diversas formas de prevención. Por lo tanto, el objetivo de este estudio fue identificar las características sociodemográficas/clínicas de usuarios de un Centro de Rehabilitación de adicciones y establecer posibles asociaciones entre perfiles de personalidad y droga de impacto.

\section{MÉTODO}

\section{Diseño de investigación}

Se trabajó desde un enfoque cuantitativo. El diseño del estudio fue de tipo no experimental, descriptivo, correlacional, ex post facto (Montero \& León, 2007), en el cual se estudió el perfil sociodemográfico y clínico de dependientes de drogas.

\section{Participantes}

La investigación se llevó a cabo de manera indirecta en una muestra por conveniencia; se realizó la revisión de 300 expedientes clínicos de usuarios que acudieron a internamiento en un Centro de Rehabilitación al noreste de México, que opera bajo una modalidad mixta de tratamiento. Los criterios de selección fueron: a) haber recibido tratamiento de rehabilitación de adicciones del 2011 al 2016; b) cumplir con los criterios diagnósticos del DSM-IV para la dependencia de sustancias, manifestado por un patrón de consumo desadaptativo que causa malestar significativo, presentar al menos tres de las siguientes condiciones: tolerancia, síntomas de abstinencia, uso de la sustancia en cantidades mayores o por tiempo más prolongado que el planeado, deseo constante por el consumo y fracasos al controlarlo, disminución de actividades laborales, sociales o lúdicas, empleo de mucho tiempo para obtener la sustancia, y uso a pesar de las consecuencias negativas psicológicas y físicas (APA, 2002).

\section{Instrumentos}

a) Cuestionario de evaluación de variables sociodemográficas, psicológicas y de salud de usuarios en rehabilitación de adicciones. Conformado por 64 ítems, distribuidos en las siguientes áreas: datos sociodemográficos (12 ítems), tratamiento (siete ítems), historia de consumo (32 ítems), información médica (dos ítems) y aspectos psicosociales (14 ítems). Las opciones de respuesta son abiertas, dicotómicas o tipo Likert. El instrumento se construyó ad hoc, con la finalidad de obtener la información más relevante de los expedientes clínicos. Los datos de este cuestionario se completaron a partir de dicho expediente.

b) Inventario Multifásico de la Personalidad Minnesota (MMPI-2; Hathaway \& Mckinley, 1995), estandarización mexicana (Lucio, Reyes-Lagunes, \& Scott, 1994). Cuestionario dirigido a adultos que consta de 567 reactivos con respuestas dicotómicas (verdadero/falso), utilizado para elaborar perfiles psicológicos e identificar características psicopatológicas de la personalidad. De acuerdo con Lucio, Pérez y Ampudia (1997), la confiabilidad alfa ha resultado adecuada para la población mexicana: escalas clínicas básicas (.66), escalas de contenido (.67), suplementarias (.73).

c) Personality Disorder Questionnaire PDQ-4+ (Hyler, 1994). Cuestionario de auto aplicación, integrado por 100 ítems de respuesta falso/verdadero, acordes con los criterios diagnósticos del DSM-IV-TR para los trastornos de personalidad. Este instrumento se ha traducido a diversos idiomas y se utiliza con frecuencia en la práctica clínica. En México se cuenta con la traducción de López Ramírez y Chávez León (2012) y se ha reportado su uso en diversos estudios (Benítez Camacho, Chávez-León, Ontiveros Uribe, Yunes Jiménez, \& Náfate López, 2010; López Ramírez \& Chávez León, 2012).

Para efectos de esta investigación se conformaron perfiles de personalidad de acuerdo con la categorización del DSM-IV-TR (APA, 2002). El psicólogo evaluador del centro realizó una integración diagnóstica de los resul- 
tados del MMPI-2 y PDQ-4+, a partir de los cuales se identificaron los perfiles de personalidad paranoide, esquizoide, esquizotípico, antisocial, límite, histriónico, narcisista, evitativo, dependiente y obsesivo-compulsivo. Finalmente, estos perfiles fueron confirmados por la evaluación clínica del psiquiatra de la institución.

\section{Procedimiento}

La información se obtuvo de los archivos del Centro de Rehabilitación de adicciones. Para el acceso se contó con la autorización de los directivos, se firmó carta de confidencialidad por parte del investigador, en la cual se estableció el compromiso de utilizar los datos con fines meramente de investigación, a fin de conservar el anonimato y señalando que dicho proceso no representaba daño para los participantes.

\section{Análisis estadísticos}

Después de la obtención de los datos y su captura en el programa estadístico SPSS versión 21, se realizaron análisis descriptivos: medidas de tendencia central y dispersión. Para el análisis inferencial se utilizó el estadístico $X^{2}$ para valorar las asociaciones entre perfiles de personalidad y droga de impacto.

\section{RESULTADOS}

Respecto a los datos sociodemográficos, una mayor cantidad de hombres ha ingresado a tratamiento del 2011 al 2016. La muestra estuvo conformada mayormente por estudiantes de bachillerato y licenciatura (Tabla 1).

Con relación al tratamiento, $44.3 \%(n=133)$ refirió haber buscado apoyo o atención en otros centros de rehabilitación antes de su internamiento; de ellos, 8.6\% $(n=26)$ indicó haber pedido ayuda en más de cuatro ocasiones; $64.3 \%(n=193)$ de la muestra recibió un tratamiento de 45 a 60 días y $64.3 \%(n=193)$ indicó usar drogas combinadas (policonsumo).

$57.4 \%$ de los participantes $(n=172)$ reportó el alcohol como su droga de inicio; los usuarios indicaron haber comenzado a utilizar sustancias en una media de edad de 15.21 años $(D T=4.183)$, hasta $46.7 \%(n=140)$ reportó seguir consumiendo su droga de inicio más de tres veces al día.

Las principales drogas de elección (droga de impacto) reportadas por esta muestra fueron alcohol (39\%), mariguana (26.3\%) y cocaína (17.7\%; Tabla 2). La mayor parte de los usuarios inició el consumo de su droga de elección entre los 11 y los 20 años de edad y reportaron continuar consumiéndola más de tres veces por día en la actualidad.

Tabla 1

Características individuales de la muestra

\begin{tabular}{|c|c|c|c|}
\hline Características & $(n=300)$ & Características & $(n=300)$ \\
\hline Sexo & $\%$ & Principal actividad & $\%$ \\
\hline Hombres & 83.7 & Estudiante & 13 \\
\hline Mujeres & 16.3 & Actividad laboral & 41 \\
\hline Estado civil & & Desempleado & 41.7 \\
\hline Soltero & 54.7 & Labores del hogar & 1.7 \\
\hline Casado & 20 & Pensionado & 2.7 \\
\hline Unión libre & 13 & Policonsumo & \\
\hline Separado & 7.7 & Sí & 64.3 \\
\hline Divorciado & 4.3 & No & 35.7 \\
\hline Viudo & 0.3 & Estatus de tratamiento & \\
\hline Nivel escolar & & Concluido & 58.3 \\
\hline Sin estudios & 1.3 & No concluido & 41.7 \\
\hline Primaria & 8 & Seguimiento a seis meses & \\
\hline Secundaria & 20.3 & Sin seguimiento & 41.3 \\
\hline Preparatoria & 36.7 & En abstinencia & 43.3 \\
\hline Estudios técnicos & 2.3 & En consumo & 15.3 \\
\hline Estudios superiores & 30.7 & & \\
\hline Posgrado & 0.7 & & \\
\hline
\end{tabular}


Tabla 2

Principales drogas de impacto

\begin{tabular}{lcc}
\hline Sustancia de impacto & Frecuencia & Porcentaje \\
\hline Alcohol & 117 & 39 \\
Mariguana & 79 & 26.3 \\
Cocaína & 53 & 17.7 \\
Inhalables & 32 & 10.7 \\
Otras & 19 & 6.3 \\
\hline
\end{tabular}

El 99\% ( $n=297)$ de la muestra indicó haberse realizado recientemente la prueba del VIH y conocer su resultado (debido a los procedimientos de ingreso de la clínica donde se realizan los estudios con el consentimiento del usuario).

Dentro de las enfermedades médicas, la anemia (12.3\%) y la hepatitis alcohólica (9.7\%) fueron las más frecuentes. Cabe señalar que 52\% $(n=166)$ de la muestra presentó otros problemas mentales en comorbilidad con su dependencia, entre las que destacan el trastorno de ansiedad generalizada (10.3\%) y el trastorno depresivo mayor (9.3\%). También se encontró que los usuarios presentaban otros problemas de atención clínica de tipo psicosocial, entre ellos problemas de relación con los padres $(30.7 \%)$, ruptura familiar por separación o divorcio $(25.3 \%)$ y relación conflictiva con el conyugue o pareja (14.7\%), haber sido afectado en la niñez por una relación parental conflictiva y duelos no complicados (6.3\%; Tabla 3).

Se encontró mayor cantidad de usuarios con perfiles de personalidad antisocial (21\%), seguidos por rasgos de personalidad límite (16.7\%) y esquizoide (13.7\%). Se utilizó la prueba $X^{2}$ para conocer la relación entre droga de impacto y perfiles de personalidad; se encontraron como datos importantes una relación significativa en el perfil de personalidad antisocial de 63 usuarios y el consumo de mariguana $\left(X^{2}=12,020 ; g l=4 ; p=.017\right.$ ) y una relación tendiente a la significación estadística entre el perfil evitativo de 15 usuarios y el consumo de alcohol $\left(X^{2}=8,779 ; g l=4, p=.067\right.$; Tabla 4).

Respecto a la familia de origen, $62.7 \%(n=188)$ indicó provenir de una familia tradicional (padre, madre e hijos), $24 \%$ ( $n=72$ ) de una monoparental y $13.3 \%(n=40)$ reconstruida. $27.3 \%$ de los usuarios señaló haber formado una familia nuclear de tipo tradicional $(n=82), 24.3 \%$ monoparental $(n=73)$ y $3 \%$ reconstruida $(n=9) ; 52 \%(n$ = 156) reportó haber tenido padre alcohólico.

Un porcentaje considerable (21\%) reportó haber sido víctima de abuso sexual, principalmente por desconocidos. Casi la mitad de la muestra reportó haber tenido ideas suicidas, $20.3 \%(n=61)$ ha planeado un suicidio, pero hasta $26 \%(n=78)$ ha intentado suicidarse. $43 \%$ $(n=130)$ de la muestra reportó mantenerse en abstinencia a seis meses de seguimiento.

\section{DISCUSIÓN Y CONCLUSIONES}

En esta investigación la muestra estuvo conformada en su mayoría por hombres solteros, lo que coincide con

Tabla 4

Perfil psicológico

\begin{tabular}{lcc}
\hline & Frecuencia & Porcentaje \\
\hline Perfil de personalidad antisocial & 63 & 21 \\
Perfil de personalidad límite & 50 & 16.7 \\
Perfil de personalidad esquizoide & 41 & 13.7 \\
Perfil de personalidad & 35 & 11.7 \\
esquizotípica & 21 & 7 \\
Perfil de personalidad paranoide & 15 & 5 \\
Perfil de personalidad evasiva & 12 & 4 \\
Perfil de personalidad narcisista & & 1.3 \\
Perfil de personalidad obsesiva- & 4 & \\
compulsiva & & 1 \\
Perfil de personalidad & 3 & 1 \\
dependiente & 3 & \\
Perfil de personalidad histriónica & & \\
\hline
\end{tabular}

Tabla 3

Comorbilidades

\begin{tabular}{|c|c|c|c|c|c|}
\hline Enfermedades médicas & Frecuencia & $\%$ & Trastornos mentales & Frecuencia & $\%$ \\
\hline Anemia & 37 & 12.3 & Trastorno de ansiedad generalizada & 31 & 10.3 \\
\hline Hepatitis alcohólica & 29 & 9.7 & Trastorno depresivo mayor & 28 & 9.3 \\
\hline Trigliceridemia & 16 & 5.3 & Trastorno de control de impulsos & 10 & 3.3 \\
\hline Dislipidemia & 12 & 4 & Esquizofrenia & 6 & 2 \\
\hline Hipertensión & 10 & 3.3 & Trastorno por estrés postraumático & 2 & 0.6 \\
\hline VIH & 3 & 1 & Trastorno bipolar & 2 & 0.6 \\
\hline
\end{tabular}


datos nacionales (Secretaría de Salud, 2016). Aunque las mujeres acuden en menor proporción, la Encuesta Nacional de Consumo de Drogas, Alcohol y Tabaco (ENCODAT) 2016-2017 (Villatoro-Velázquez et al., 2017a) indica que existe un incremento importante en el uso de drogas en la mujeres. Es necesario trabajar en el desvanecimiento del estigma social para favorecer el reconocimiento de las adicciones en la mujer y brindar apoyos sociales formales para su tratamiento, pues es sabido que las mujeres con un trastorno adictivo pueden tener trastornos depresivos, de ansiedad o personalidad, y la progresión de la enfermedad en ellas ocurre con mayor celeridad (Souza, M. \& Cruz, M. 2009).

En México se ha reportado que en los centros de tratamiento la droga de inicio suele consumirse a una edad promedio de 14.7 años y $91.9 \%$ de los usuarios refiere seguir consumiéndola en la actualidad (Secretaría de Salud, 2016); estos datos se asemejan a lo aquí encontrado, donde la media de edad de consumo por primera vez fue de 15.21 años y coincide con lo reportado en otros estudios (Capistrano et al., 2013; Cázares López et al., 2010; Oropeza et al., 2013). La adolescencia representa una etapa crítica en la que múltiples factores de riesgo juegan un papel decisivo para el inicio del consumo; de esta forma, un joven que empieza a consumir a más temprana edad tiene mayor riesgo de generar dependencia (NIDA, 2016)

Las principales drogas de impacto reportadas en esta investigación fueron: alcohol, mariguana y cocaína, esto coincide con lo reportado por eI SISVEA (Secretaría de Salud, 2016); y similar a lo reportado en otros estudios con muestras afines (Cázares López et al., 2010; Souza \& Cruz, 2009); los resultados sobre el consumo de cocaína difieren de los de Diaz-Negrete et al., (2015), ellos estudiaron las características sociodemográficas de solicitantes a tratamiento ambulatorio de 116 unidades de Centros de Integración Juvenil, donde el consumo de cocaína y crack presentó una tasa media de crecimiento anual negativa en el 2014 en la zona Nororiental (Tamaulipas, Nuevo León y San Luis Potosí). Aunque existen diferencias en las muestras, llama la atención que en solicitantes de tratamiento ambulatorio de la zona Norte la demanda por cocaína haya disminuido; sin embargo, en una muestra de pacientes en rehabilitación sea la cocaína una de las principales drogas de impacto, lo que sugiere la necesidad de tratamiento más intensivo de estos usuarios por el fuerte poder adictivo de la sustancia.

Los padecimientos médicos que sobresalieron fueron la anemia y la hepatitis; otros estudios han reportado presencia de padecimientos clínicos y enfermedades crónicas en muestras similares (Cázares et al., 2010; Capistrano et al., 2013). Respecto a los perfiles de personalidad, en esta muestra se encontraron con mayor frecuencia perfiles de personalidad antisocial y límite, similar a estudios como el de Bricolo, Gomma, Bertani y Serpelloni (2002) y Fernández-Montalvo et al. (2004), quienes encontraron una alta presencia de trastorno antisocial de la personalidad en una comunidad terapéutica. En México, López Ramírez y López del Castillo (2007) reportaron una asociación significativa entre alcoholismo y presencia de trastorno antisocial de la personalidad, histriónico, esquizoide, límite, negativista, esquizotípico y depresivo.

En este estudio se encontró la presencia de trastornos de ansiedad generalizada y depresión. De acuerdo con San et al. (2016), los usuarios con trastornos de ansiedad son proclives a desarrollar trastornos por abuso de sustancias, por lo cual es un objetivo relevante su diagnóstico y tratamiento. También mencionan que los trastornos por uso de sustancias y los trastornos depresivos presentan comorbilidad frecuentemente y tienen en común factores de riesgo como acontecimientos vitales estresantes, traumas psicológicos, alteraciones neurobiológicas y vulnerabilidad genética. Los hallazgos en esta muestra sobre la presencia de trastornos mentales en los pacientes internos llevan a pensar en el tema de la patología dual (presencia de trastornos psiquiátricos en pacientes con trastornos por uso de sustancias), tema que requiere mayor profundización en América Latina.

La Organización Panamericana de la Salud (OPS), la Delegación del Gobierno para el Plan Nacional de Drogas (DGPNSD), la Sociedad Española de Trastornos Duales (SEPD) y el Instituto Nacional de Psiquiatría Ramón de la Fuente Muñiz (INPRFM) han generado la Iniciativa Panamericana para la Patología Dual, en la cual se proponen cuatro líneas de acción para la mejora de los servicios de salud: consensuar el concepto de patología dual, proponer un modelo de tratamiento, crear entrenamiento basado en competencias, y crear redes para la investigación sobre patología dual (Alfonzo-Bello et al., 2017), por lo que se sugiere ampliar este tema en futuros estudios que ayuden a caracterizar de manera más completa a los usuarios que acuden a solicitar tratamiento especializado.

Dentro de otros problemas de atención clínica, aparecen en esta muestra con mayor frecuencia problemas de relación entre padres e hijos, caracterizados por comunicación inadecuada, dificultades con la disciplina y sobreprotección, lo cual contribuye a la aparición de síntomas clínicos en padres y/o hijos, según el DSM-5 (APA, 2014). Iraurgi Castillo, Sanz Vázquez y Martínez Pampliega (2004) exponen que la familia es una variable importante en el inicio y el mantenimiento del consumo de sustancias; el uso de drogas y una peor situación médica y psiquiátrica se asociaron positivamente con estrés familiar y baja satisfacción familiar, peor comunicación y 
recursos familiares. Otro estudio realizado en México por López Ramírez y López del Castillo (2007) encontró que los pacientes alcohólicos presentaban un promedio mayor de conductas psicopatológicas en la niñez, como conductas disociales, angustia por separación, conductas oposicionistas, desacato a reglas, trastorno por déficit de atención y problemas con la autoridad.

La mitad de la muestra mencionó tener un padre con alcoholismo. Ruiz, Herrera, Martínez, y Supervielle (2014) comentan que el antecedente de consumo de la figura paterna representa un factor de riesgo para el inicio del consumo de sustancias en la adolescencia. Estos datos enmarcados en el estudio de las familias con consumo de sustancias llevan a pensar en un tema ya expuesto en la literatura científica, grupos de autoayuda para familiares de alcohólicos Al-Anon y en la práctica clínica: el término denominado codependencia (Noriega Gayol \& Ramos Lira, 2002). Una persona codependiente es quien se ha afectado de manera significativa por una relación actual o pasada surgida en un ambiente familiar fuertemente estresante por el consumo de sustancias, muestra síntomas como vergüenza, miedo, culpa, negación, confusión, rigidez y dificultades con la formación de la autoestima (Potter-Efron \& Potter-Efron, 1989); si bien este término se ha utilizado principalmente en mujeres parejas de pacientes adictos (Noriega Gayol \& Ramos Lira, 2002), cabe considerar estudiarlo en dependientes de sustancias, para conocer las afectaciones generadas a partir de la convivencia con otro familiar alcohólico, especialmente de la figura paterna.

Una quinta parte de la muestra estudiada reportó haber sufrido abuso sexual principalmente por desconocidos. Al respecto, Pérez y Mestre (2013) exponen que existen evidencias de que el abuso sexual infantil, principalmente en mujeres, se relaciona con el padecimiento de dependencia de sustancias en la vida adulta.

Una cuarta parte de la muestra presentó intentos suicidas. Ocampo, Bojórquez, y Cortés (2009) encontraron en su estudio epidemiológico que estar bajo los efectos de sustancias fue detonador de suicidio, a mayor número de sustancias detectadas en hombres y mujeres, la posibilidad de suicidio se incrementó, principalmente en las mujeres.

Como limitantes de este estudio hay que tomar en cuenta que los resultados no son representativos de la población general; el alcance del estudio fue meramente descriptivo-correlacional, buscando caracterizar a los usuarios de una institución especializada en tratamiento de adicciones. Como limitación metodológica, se recolectó la información de manera indirecta a través de los expedientes clínicos, por lo tanto, como conclusiones se recomienda continuar investigando de manera directa las barreras y dificultades de las mujeres con adicciones para recibir tratamiento, profundizar el tema de la comorbilidad, siguiendo los lineamientos de la Iniciativa Panamericana para trastornos duales, y continuar con acciones de prevención primaria desde la infancia, y prevención secundaria en población de estudiantes de bachillerato y licenciatura. La rehabilitación es una opción favorable y necesaria para la recuperación de los usuarios y sus familias.

\section{FUENTES DE FINANCIAMIENTO}

La presente investigación no fue financiada por ningún organismo ni institución.

\section{CONFLICTOS DE INTERÉS}

Los autores manifiestan que no existe ningún conflicto de interés en la realización del manuscrito.

\section{AGRADECIMIENTOS}

Se expresa un agradecimiento a la Secretaría de Salud del estado de Tamaulipas y al Centro de Internamiento y Rehabilitación (RENACER), a la licenciada Matilde Torres Herrera y a la licenciada Rosa Elia Barrón Álvarez, por las facilidades otorgadas para el desarrollo de este trabajo.

\section{REFERENCIAS}

Alfonzo-Bello, L., Szerman, N., Marín-Navarrete, R., Arribas-lbar, E., .... Pérez-López, A. (2017). Ibero-American initiative for dual disorders to improve public treatment programs. Salud Mental, 4O(6), 249-255. doi:10.17711/SM.0185-3325.2017.032

American Psychiatric Association. (2002). DSM-IV-TR: Manual Diagnóstico y Estadístico de los Trastornos Mentales. Barcelona: Masson.

American Psychiatric Association. (2014). Manual diagnóstico y estadístico de los trastornos mentales (DSM-5). Madrid: Editorial Médica Panamericana

Benítez Camacho, E., Chávez-León, E., Ontiveros Uribe, M. P., Yunes Jiménez, A., \& Náfate López, O. (2010). The levels of psychological functioning of personality and the mechanisms of defense. Salud Mental, 33(6), 517-526.

Bricolo, F., Gomma, M., Bertani, M., \& Serpelloni, G. (2002). Prevalencia de trastornos de personalidad en una muestra de 115 clientes con trastornos por uso de drogas. Adicciones, 14(4), 491-496. doi: 10.20882/adicciones.480

Capistrano, F. C., Ferreira, A. C. Z., Silva, T. L., Kalinke, L. P., \& Maftum, M. A. (2013). Perfil sociodemográfico e clínico de dependentes químicos em tratamento: análise de prontuários. Escola Anna Nery Revista de Enfermagem, 17(2), 234-241. doi 10.1590/S1414-81452013000200005

Cardenal, V., Sánchez, M. P., \& Ortiz-Tallo, M. (2007). Los trastornos de personalidad según el modelo de Millon: una propuesta integradora. Clínica y salud, 18(3), 305-324. 
Carou, M., Romero, E., \& Luengo, M. A. (2013). Patrones de consumo y variables de personalidad en drogodependientes a tratamiento. Revista española de drogodependencias, (38)3, 217-232.

Cázares López, M. J., González-Menéndez. A., Torres-Lobo, M., Secades-Villa, R., Fernández-Hermidia, J. R., \& Álvarez, M. M. (2010). Comparación del perfil psicopatológico y adictivo de dos muestras de adictos en tratamiento: en prisión y en comunidad terapéutica. International Journal of Clinical and Health Psychology, 10(2), 225-243.

Díaz-Negrete, D. B., Gutiérrez-López, A. D., Fernández-Cáceres, C., \& Sánchez-Huesca, R. (2015). Consumo de sustancias y características sociodemográficas de solicitantes de tratamiento ambulatorio en una red de atención especializada en México; análisis comparativo entre 2007 y 2014. Revista Internacional de Investigación en Adicciones, 1(1), 16-26. doi: 10.28931/ riiad.2015.1.03

Fernández-Montalvo, J., López Goñi. J. J., Landa, N., Illescas, C., Lorea, I., \& Zarzuela, A. (2004). Trastornos de personalidad y abandonos terapéuticos en pacientes adictos: resultados en una comunidad terapéutica. International Journal of Clinical and Health Psychology, 4(2), 271-283.

Hathaway, S. R., Butcher, J. N., \& Mckinley, J. C. (1995). Inventario Multifásico de la Personalidad Minnesota-2. México: El Manual Moderno.

Hyler, S. E. (1994). PDQ-4+ Personality Diagnostic Questionnaire-4+. New York: New York State Psychiatric Institute.

Iraurgi Castillo, I., Sanz Vázquez, M., \& Martínez Pampliega, A. (2004). Funcionamiento familiar y severidad de los problemas asociados a la adicción a drogas en personas que solicitan tratamiento. Adicciones, 16(3), 185-195. doi: 10.20882/adicciones. 400

López Ramírez, M. E., \& Chávez León, E. (2012). Relación de los mecanismos de defensa y los trastornos de la personalidad. Revista Latinoamericana de Psiquiatría, 11(3), 73-81.

López Ramírez, M. E., \& López del Castillo Esparza, A. (2007). Relación del alcoholismo con los trastornos de la personalidad en una muestra mexicana. Revista Electrónica de Psicología Iztacala, 10(1), 1-15.

Lucio, G. M. E., Pérez, J. M., \& Ampudia, A. (1997). Un estudio de confiabilidad test-retest del MMPI-2 en un grupo de estudiantes mexicanos. Revista Mexicana de Psicología, 14(1), 55-62.

Lucio, G. M. E., Reyes-Lagunes, I., \& Scott, R. L. (1994). MMPI2 for Mexico: Translation and adaptation. Journal of Personality Assessment, 63(1), 105-116. doi: 10.1207/s15327752jpa6301_9

Montero, I., \& León, O. G. (2007). A guide for naming research studies in Psychology. International Journal of Clinical and Health Psychology, 7(3), 847-862.

National Institute on Drug Abuse. (2016). Entendiendo el uso de drogas y la adicción. Recuperado de: https://www.drugabuse. gov/es/publicaciones/drugfacts/entendiendo-el-uso-de-drogasy-la-adiccion.

Noriega Gayol, G., \& Ramos Lira, L. (2002). Construcción y validación del instrumento de codependencia (ICOD) para las mujeres mexicanas. Salud Mental, 25(2), 38-48.
Ocampo, R., Bojórquez, I., \& Cortés, M. (2009). Consumo de sustancias y suicidios en México: resultados del Sistema de Vigilancia Epidemiológica de las Adicciones, 1994-2006. Salud Pública de México, 51(4), 306-313.

Oficina de las Naciones Unidas Contra la Droga y el Delito. (2017). Informe Mundial sobre las Drogas 2017. Viena, Austria: UNODC. Recuperado de: https://www.unodc.org/wdr2017/field/WDR_ Booklet1_Exsum_Spanish.pdf

Oropeza, T. R., Lira, M. J., \& Rosales, G. M. (2013). Perfil sociodemográfico y patrones de consumo de drogas de pacientes de cocaína y crack. Diversitas, 9(2), 373-382. doi: 10.15332/s17949998.2013.0002.10

Pérez, D. R. F., \& Mestre, G. M. (2013). Abuso sexual en la infancia y la drogodependencia en la edad adulta. Papeles del Psicólogo, 34(2), 144-149.

Potter-Efron, R. T., \& Potter-Efron, P. (1989). Assessment of co-dependency with individuals from alcoholic and chemically dependent families. Alcoholism Treatment Quarterly, 6(1), 37-57. doi:10.1300/j020v06n01_04

Ruiz, C. H., Herrera, B. A., Martínez, B. A., \& Supervielle, M. M. (2014). Comportamiento adictivo de la familia como factor de riesgo de consumo de drogas en jóvenes y adolescentes adictos. Revista Cubana de Investigaciones Biomédicas, 33(4), 402409.

San, L., Arranz, B., Arrojo, M., Becoña, E., Bernardo, M., Caballero, L., ... Zorrilla, I. (2016). Clinical guideline for the treatment of dual pathology in the adult population. Adicciones, 28(1), 3-5. doi: 10.20882/adicciones.784

Secretaría de Salud (2009). Norma Oficial Mexicana NOM-028SSA2-2009 Para la prevención, tratamiento y control de las adicciones. Recuperado de: http://www.conadic.salud.gob.mx/pdfs/ norma_oficial_nom.pdf

Secretaría de Salud (2016). Sistema de Vigilancia Epidemiológica para las Adicciones. Informe 2016. Recuperado de: http://187.191.75.115/gobmx/salud/documentos/info_sisvea/ informes_sisvea_2016.pdf

Souza, M. M., \& Cruz, M. D. L. (2009). La patología adictiva de la mujer y su comorbilidad. Revista de la Facultad de Medicina UNAM, 52(1), 23-29.

Villatoro-Velázquez, J. A., Reséndiz-Escobar, E., Mujica-Salazar, A., Bretón-Cirett, M., Cañas-Martínez, V., Soto-Hernández. I., ... Mendoza-Alvarado. L. (2017a) Encuesta Nacional de Consumo de Drogas, Alcohol y Tabaco 2016-2017: Reporte de Drogas. Ciudad de México, México: INPRFM. Disponible en: https://drive. google.com/file/d/1zIPBiYB3625GBGIW5BX0TT_YQN73eWhR/ view

Villatoro-Velázquez. J. A., Resendiz-Escobar, E., Mujica-Salazar, A., Bretón-Cirett, M., Cañas-Martínez, V., Soto-Hernández. I., ... Mendoza-Alvarado. L. (2017b) Encuesta Nacional de Consumo de Drogas, Alcohol y Tabaco 2016-2017: Reporte de Alcohol. Ciudad de México, México: INPRFM. Disponible en: https://drive.google.com/file/d/1rMIKaWy34GR51sEnBK2u2q_BDK9LA0e /view 